\title{
Gamers' Cognitive Process on Gameplay in Massively Multi-player Online Role- Playing Game: Towards World of Warcrafts Players' the Difference
}

\author{
Seungkeun Song, Joohyeon Lee \\ Cognitive Science Program, The Graduate \\ School, Yonsei University, 134 Shinchondong, \\ Seodaemungu, Seoul 120-749, Korea \\ \{songsk,ljhyeon\}@yonsei.ac.kr
}

\begin{abstract}
The objective of this study is to explore the differences according to gamers' expertise: experts, intermediate gamers, and novices. To this end, this study proposes an analytic framework that focuses on the gamers' cognitive process. An empirical experiment within the domain of MMORPG game was conducted where verbal protocols and action protocols were collected. The results of the study reveal that the different behavior patterns result from standardizing of their actions for experts and forming the specific patterns of action for intermediate game players. The gameplay pattern was elucidated by much more time and steps in the game for novices than for intermediate gamers. While there was an intensive distribution in the early stage of the game for experts, the novices' behavior pattern appeared in a divergent distribution in the whole gameplay. This study is concluded with key implications to support game design to improve the quality of game product.
\end{abstract}

Keywords: MMORPG, Game Design, Protocol Analysis, Problem Solving, Play Theory, Analytic Framework

\section{Introduction}

Some people have built up some prejudices against the computer games. First, the computer games have been seen as children's medium. This means that they are easily belittled as insignificant-something that will be 'grown out of'- and requiring no research. This is empirically wrong because people who played the computer games when young rise to the main stream when we examine an aspect of the game market extension [1]. Second, the computer games have been considered simple trifles (i.e., low art) carrying none of the weight, gravitas or credibility of more traditional media. Consequently, they have been unfairly compared with respectable media like film [2]. Moreover, the computer games have been considered a lack of seriousness in the treatment of themselves due to "restart" function. Although a gamer was dead to make mistake during the gameplay, it has been seen as being relatively insignificant because they can restart at 'save point'

\author{
Jun Jo \\ Robotics and Games Laboratory, PMB50 Gold \\ Coast Mail Centre, Griffith University, \\ Gold Coast Campus, Queensland, Australia \\ $\{j . j o\} @ g r i f f i t h . e d u . a u$
}

where the gamers may play the game again [3]. Academics, therefore, have ignored the computer games because of these prejudices [4].

However, the latest computer games are big business and growing up as movie industry in terms of its size. In 1996, US computer and video game software sales totaled about 3.5 billion dollars. By 2004, a mere eight years later, software sales had more than doubled, generating an income of $\$ 7.3$ billion. [5]. Because of the rapid growth of the industrial size and popularity, the computer games have become a central axis of a popular culture that is not to be sneezed at. Furthermore, So far, various effects of the computer games have been discussed. US psychologists have argued whether the computer games will be harmful or not since 1980. This discussion has continued until now [6]. When considering the endless dispute and the rapid growth in entertainment industry, we should take into account seriously the computer games

During the last few years, a debate took place within the game scholar community, a debate that opposed two groups: ludologists and narratologists. Ludologists are supposed to focus on game mechanics and reject analyzing games as the extension of the narrative, while narratologists argue that games are closely connected to stories. While they made computer studies futile due to ever-going discussions regarding the identity of the computer games, little empirical research has been conducted specifically on the property of the computer games itself and game structure in terms of the gameplay [7]. This study holds a critical mind against their unproductive debate of the computer games and tries to empirically study gamers' playing experience rather than external domain of games.

The goals of this study, therefore, are to (1) propose an analytic framework for the gameplay (2) explore the difference between players according to their expertise: experts, intermediate gamers, and novices.

\section{Literature review}

\subsection{Gameplay in play theory}

The two most influential sources for definitions of gameplay are the more general definitions of play offered by Huizinga [8] and Caillois [9]. For Huizinga, the main 
characteristics of play are : that it is a voluntary activity, freely entered into as a source of pleasure and "never imposed by physical necessity or moral duty"; that it involves a stepping out of " real" life into "a temporary sphere of activity with a disposition all of its own"; that this occurs in its own distinct time and place, ordered and rule-bound; and play tends to create its own communities, on the basis of particular shared activities [8]. Caillois' definition is broadly similar, building on Huizinga and adding some extra ingredients. For Caillois, play is essentially free. It is separate, and activity "circumscribed within limits of time and space, defined and fixed in advance," governed by rules, and make-believe. To these dimensions Caillois adds the suggestion that play is uncertain, the precise course and result not being determined in advance "and some latitude for innovations being left to the player's initiative," and unproductive, "creating neither goods nor wealth" [9]. According to Caillois [9], Paidea is "prodigality of physical or mental activity which has no immediate useful objective, nor defined objective, and whose only reason to be is based in the pleasure experimented by the player" in play based on its property. Ludus is defined as an "activity organized under a system of rules that defines a victory or a defeat, a gain or a loss." Moreover, he has classified the category of the game into competition (Agon), chance (Alea), simulation (Mimicry), and vertigo (Ilinx) according to existence and non existence of player's will and rule. When examining four types of play in aspects of MMORPG game, it may trigger competition (Agon) to increase accomplishment desire through the quest conduction given by a specific mission. In the case of the items offered by the hunting monster, gamers may have the expectation of the chance (Alea): what item will be provided among randomly assigned items. Moreover, gamers may identify themselves as an agent living in a game environment. Consequently, MMORPG game which is played through the medium of name, sex, and job with the third person character may correspond to simulation (Mimicry). Finally, transportation as Wyverns (Windriders) from a certain town to another town may be accompanied with vertigo (Ilinx). Although Huizinga and Caillois did not experience a computer game age, its property and category in play theory may be applicable in doing research the gameplay in MMORPG game.

\subsection{Gameplay as problem solving}

In general, the reason why gamers play the game is to accomplish given goals [10]. If that was the case what does the gamer's goal to achieve mean? We can understand it from 'Problem Solving Theory': the achievement of gamer's goal though the gameplay is viewed as a search process in problem spaces using operators within constraints [11]. Specifically, computer games may provide gamers with various goals to accomplish when they play the game. The computer game may present virtual space where various activities are available for the goal accomplishment to gamers. It may provide them with some kind of tasks to surely solve within it. Moreover, the computer game may offer gamers operators related to the task as some kind of game interface to conduct it during the gameplay. Finally, gamers can explore the rule of the game embedded in the computer games by designers when they accomplish the goal using operators during the gameplay. Gamers, therefore, are possessed by various goals during the gameplay. They should behave in order to achieve their goals while complying with constraints in the problem space corresponding to the game world.

\subsection{Related research for building an analytic framework}

Kavakli and Thorne [12] have modeled cognitive process in the gameplay by the extension of study on architects' cognitive model [13]. They have utilized the protocol analysis based on content-oriented approach for doing research on what gamers see, attend to, think of, and retrieve from memory while playing an adventure game. While their research can help understand gamers' emotion during the gameplay, it is difficult to derive dynamic information processes such as human's problem solving, reasoning, decision making. Consequently, the protocol analysis should require a complementary method that combines process-oriented approach with contentoriented approach to understand the gameplay deeply. Kim et. al. [14] proposes a cognitive framework describing the software development process in objectoriented design (OOD) as building internal representations and developing rules. Kim's study was a definite complement to Kavakli's study because Kim et. al. clarify the cognitive process with which Kavakli and Thorne dealt insufficiently. As another process-oriented approach, Bonnie John and Alonso Vera [15] used GOMS to analyze interaction with computer games. Their work represented an extension of the GOMS methodology to much more interactive, machine-paced, and graphic-based applications than has been previously demonstrated as editing task or telephone operator's call handling. Their GOMS analysis predicted about $60 \%$ of the behavior at the keystroke-level operators with functional-level operators predicting close to $100 \%$ for expert's behavior pattern. Therefore, they presented the possibility to apply GOMS methodology to computer game study.

In summary, the play theory defined by Huizinga [8] and Caillois [9] and the problem solving theory by Newell and Simon [11] were reviewed in order to understand the characteristics of the gameplay. In addition to reviewing two theories, Kavakli's study [12] based on contentoriented approach, Kim's study [14] based on processoriented approach, and John's study [15] based on GOMS 
methodology in computer games were investigated to develop analytic framework for the gameplay.

\section{Analytic framework for gameplay}

This study proposes an analytic framework for the gameplay as follows (see Table 1).

Table 1. Analytic Framework for gameplay

\begin{tabular}{|c|c|c|c|c|c|c|}
\hline \multicolumn{2}{|c|}{ This Study } & \multicolumn{5}{|c|}{ Related Literatures } \\
\hline \multirow[b]{2}{*}{ Category } & \multirow[b]{2}{*}{$\begin{array}{l}\text { Action, } \\
\text { Content }\end{array}$} & \multicolumn{2}{|c|}{ Theories } & \multicolumn{3}{|c|}{ Studies } \\
\hline & & $\begin{array}{l}\text { Play } \\
(1944, \\
1958)\end{array}$ & $\begin{array}{c}\text { Problem } \\
\text { Solving } \\
\text { (1972) }\end{array}$ & $\begin{array}{l}\text { Kavakli, } \\
\text { Thorne } \\
(2002)\end{array}$ & $\begin{array}{l}\text { John, } \\
\text { Vera } \\
(1992)\end{array}$ & $\begin{array}{l}\text { Kim, } \\
\text { et. al. } \\
\text { (1995) }\end{array}$ \\
\hline Perceptual & $\begin{array}{c}<\text { P-action }> \\
\text { Id, Rd }\end{array}$ & & $\begin{array}{l}\text { Physical } \\
\text { Operator }\end{array}$ & $\begin{array}{l}\text { Perceptual } \\
\text { Action }\end{array}$ & & \\
\hline $\begin{array}{l}\text { Representati } \\
\text {-onal }\end{array}$ & $\begin{array}{c}<\text { R-content }> \\
\quad \text { Entity, } \\
\text { Relation, } \\
\text { Role, Goal } \\
<\mathrm{R} \text {-action }> \\
\text { Un /Const }\end{array}$ & & $\begin{array}{l}\text { Initial } \\
\text { State, } \\
\text { Goal, } \\
\text { State, } \\
\text { Constraint, } \\
\text { A Set of } \\
\text { States }\end{array}$ & $\begin{array}{l}\text { Functional } \\
\text { Action } \\
\text { Conceptual } \\
\text { Action }\end{array}$ & Goal & $\begin{array}{c}\text { Represe } \\
\text {-ntation } \\
\text { Space }\end{array}$ \\
\hline Functional & $\begin{array}{c}\text { F-action }> \\
\text { Search, } \\
\text { Battle, } \\
\text { Gather, } \\
\text { Avoid, } \\
\text { Comm. }\end{array}$ & & $\begin{array}{l}\text { Mental } \\
\text { Operator }\end{array}$ & & $\begin{array}{c}\text { Functio- } \\
\text { nal - } \\
\text { level } \\
\text { operator }\end{array}$ & \\
\hline $\begin{array}{l}\text { Methodolog } \\
\text {-ical }\end{array}$ & $\begin{array}{c}<\text { M-content }> \\
\text { Template, } \\
\text { F-N-C, } \\
\text { F-R-D, } \\
\text { Sequential, } \\
\text { Ambient, } \\
\text { Move Close, } \\
\text { Pulling, } \\
\text { <M-action> } \\
\text { Evoke, } \\
\text { Derive, Infer }\end{array}$ & $\begin{array}{c}\text { Property } \\
\text { Ludus, } \\
\text { Paidea } \\
\text { Type } \\
\text { Alea, } \\
\text { Agon } \\
\text { Mimicry, } \\
\text { Ilinx }\end{array}$ & $\begin{array}{c}\text { Mental } \\
\text { Operator }\end{array}$ & & $\begin{array}{l}\text { Method, } \\
\text { Select- } \\
\text { ion } \\
\text { Rule }\end{array}$ & $\begin{array}{l}\text { Rule } \\
\text { Space }\end{array}$ \\
\hline Simulative & $\begin{array}{c}<\text { S-action> } \\
\text { Generate, } \\
\text { Refine, } \\
\text { Evaluate }\end{array}$ & o & $\begin{array}{c}\text { Mental } \\
\text { Operator }\end{array}$ & & & $\begin{array}{l}\text { Instan- } \\
\text { ce } \\
\text { Space }\end{array}$ \\
\hline Kinematical & $\begin{array}{c}<\text { K-action }> \\
\text { Move,View, } \\
\text { Attack,Chat, } \\
\text { Acquire }\end{array}$ & & $\begin{array}{l}\text { Physical } \\
\text { Operator }\end{array}$ & $\begin{array}{c}\text { Physical } \\
\text { Action }\end{array}$ & $\begin{array}{c}\text { Keystr- } \\
\text { oke- } \\
\text { level } \\
\text { operator }\end{array}$ & \\
\hline
\end{tabular}

The first category, perceptual, refers to actions of identifying and reading visual-acoustic features of depicted elements on display. We call it a 'P-action'. The second category, representational, refers to actions of setting up his or her goals to achieve and determining entity, relation, and role on the internal representation. We call it an 'R-content'. Gamer understood and constructed the internal representation for given constraints within games on the basis of the obtained information. We call it an 'R-action'. The third category, functional, refers to actions of performing 'search', 'battle', 'gather', 'avoid', and 'communication' to accomplish his or her goals on the internal representation. We call it an 'F-action'. Gamer reconstructed the internal representation in order to achieve subgoals (i.e.,a particular kind of functional actions). Therefore, the forth category, methodological, refers to actions of evoking, deriving, and inferring his or her strategy to solve given problems. We call it an 'M-action'. Moreover, gamer explored the rule embedded in game space by designer and tried to apply his or her strategy to the gameplay. This strategy corresponds to the control knowledge: template, sequential, find-no-constraint, first-remove-danger, ambient, move-close, and pulling method. We call it an 'M-content'. The fifth category, simulative, refers to actions of generating, refining, and evaluating his or her method to solve the problem during confronting with the constraints. We call it an 'S-action'. The sixth category, kinematics, refers to actions of pressing buttons and clicking a mouse as a configurative action (i.e., real time physical feedback) through actions and content mentioned above. We call it a 'K-action'.

\section{Method \\ 4.1. Subjects}

Nine college students participated in the experiment. The subjects were classified into three groups according to their level of expertise with MMORPGs. The first level represented experts who were sufficiently experienced with WOW for more than a year and other console games as well as MMORPGs, the second level represented intermediate gamers that were experienced with MMORPGs but new to WOW games, and the third level represented novices who were new to MMORPGs. The subjects chosen for the study represent the general demographic distribution of computer gamers [5]. Mean age of the subjects is 20.36 with maximum 24 , minimum 18 , and standard deviation 1.934 .

\subsection{Task material}

This study chose MMORPG genre as task material because its genre was determined to coincide with viewing gameplay as problem solving process. Specially, among the various MMORPG titles available, we selected World of Warcrafts (WOW) for its commercial success [5] and its record of excellent playability as established by various game critics. In WOW, game progresses through quests. Quests signify another game comprised of small units (i.e., a game within a game). A novel and a play are often subdivided through the concept of chapter. In the case of computer games, stage and map are utilized to reduce tedium and to induce gamers' immersion. Moreover, the total content is divided into sub-contents by mission or quest. What gamers should do for each quest is apparent because the total game is divided into smaller units using quests in WOW. Traditional MMORPGs, before the appearance of WOW, had the problem that gamers often forgot their goal due to the endless story and a wide game space. This problem was solved through the concept of quests by WOW, which is the first time in the game industry. Furthermore, gamers may perceive the problems and challenges easily that they must face to win the game because WOW has an excellent design that allows for proper constraints per game content. These design factors in WOW are regarded as the important nature of gameplay. WOW, therefore, is 
worth studying for the gameplay in terms of problem solving theory

\subsection{Experimental procedure}

The experimental sessions were divided into five sections. First, the subjects were given instructions about the general nature of the experiment and were told that verbal protocols would be collected. Subjects were trained to "think-aloud" using two traditional tasks [16]. All subjects were asked to select only the warrior class from the human race of the Alliance, one of the player characters in the game, in order to guarantee a homogeneous experimental environment for the participants. The actual playing session lasted a hour. The researcher recorded subjects' utterance and actions during gameplay using audio and video equipment. They were asked to report while watching the videotape of their gameplay activities. Finally, the participants were debriefed by the researcher about the primary purpose of this experiment.

\subsection{Protocol analysis}

An exploratory protocol analysis was conducted to investigate gamers' cognitive processes. Both verbal and action protocols were collected for analysis so that a more complete trace of problem-solving behavior could be traced. Two important preconditions of protocol analysis are to identify an appropriate unit of analysis and to develop an objective coding system for each unit. We elected to use episodes - small self-contained phases of highly organized activities [11] - as the unit of analysis, considering the volume of protocol data from the experiment. While motor-level action was defined as simple code, functional-level action was defined as each episode based on the analytic framework for the gameplay [see table 1]. According to what subjects played during each episode, it was classified as one of the following coding schema: 'Search', 'Avoid', 'Battle', 'Communication', 'Gather Type A' (Find-No-Constraint as Template-Method), 'Gather Type B' (First-RemoveDanger as Template-Method), and 'Gather Type C' (Sequential-Method).

For each experiment we decided to conduct the protocol analysis step by step across all the subjects in order to increase reliability for nine subjects' protocol results. The steps were: transcription, verification of transcription, initial segmentation, segmentation test, episode coding, test for episode coding, and construction of 'Play Behavior Graphs'. For the protocols, a certain step was finished before the next step was started for any subject. The gameplay behavior was summarized into a 'Play Behavior Graph' (PBG), which is similar to the 'Problem Behavior Graph' (PBG) proposed by Newell and Simon. A PBG portrays problem solving activities as a series of searches in the problem space [11]. Examples of the PBG will be presented in the results section.

\section{Results}

This section presents the results of the coherences and differences of the gameplay within each group. In PBG (Play Behavior Graph), the axis of ordinate included 5 functional actions of 7 columns: 'Gather Type A', 'Gather Type B', 'Gather Type C', 'Communication', 'Avoid', 'Battle', and 'Search'. The horizontal axis contained 31 episodes for all of the subjects. It means the process of the gameplay. Time flows from left to right to show the dynamics of the gameplay process.

Figure 1 shows the coherences and differences of the gameplay between an expert and an intermediate gamer. For the coherences between two groups, all two groups concentrate on the 'gather action' among several functional actions from episode 2 to episode 14 (see ' $\mathrm{A}$ ' in Figure 1). They focused on the strategic action like the template-method and sequential-method because their actions were directly related to solving the problem to complete the quest goal. Next, they have the similar ending spots which are located within the range of episode 10 14 like ' $B$ ' in Figure 1 (Except an intermediate gamer 7). This result of this study reveals that game designers may estimate the elapsed time when the professional gamers consume the game content. Consequently, a concrete game planning is available. Finally, with the whole 31 episodes for 9 subjects, their gameplay shows high frequency in the early phase. This indicates they have the short steps for the gameplay and spend little time. For the differences between two groups, they show 'Gather Type C' in difference for the intermediate gamers at the early phase of gameplay (episode 2 6), for experts at the latter phase (episode $7 \sim 12$ ), as ' $C$ ', ' $D$ ' in Figure 1. When PBGs for the intermediate game player are examined in detail, they understood the rule of the game to change their strategies from sequential-method to template-method. In other words, they conduct the change of initial phase from sequential-method ('Gather Type C') to the template method ('Gather Type B'). On the other hand, for experts the sequential-method happened because the difficulty of the game causes them to make mistakes rather than those learning effects. As supporting evidence for these results, PBGs were drawn as the horizontal shape at the pattern of the gameplay by experts, but as the ascending curve shape by intermediate game player. These behavior patterns result from standardizing of their actions for experts and forming the specific patterns of action for intermediate game players.

Figure 2 presents the coherences and differences between the intermediate and novice group. For the coherences between two groups, all two groups make the 
A

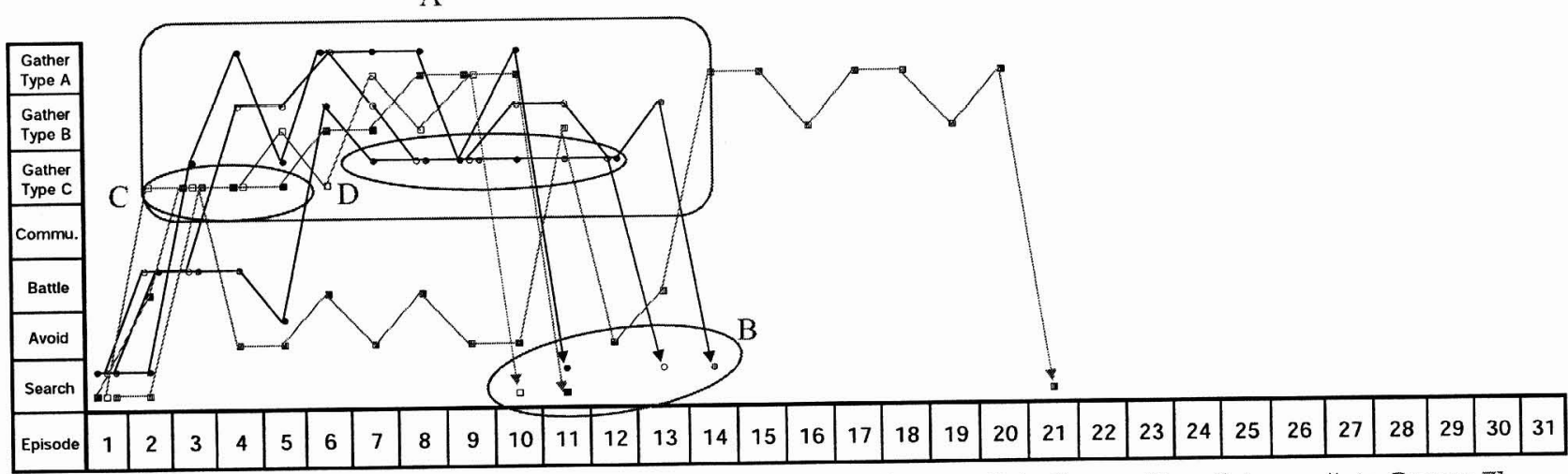

- [Expert 2], $\circ$ [Expert 8], - [Expert 1], [Intermediate Gamer 6], [Intermediate Gamer 3], 玨 [Intermediate Gamer 7]

Figure 1. Experts v.s. Intermediate gamers: The Summary of Play Behavior Graph

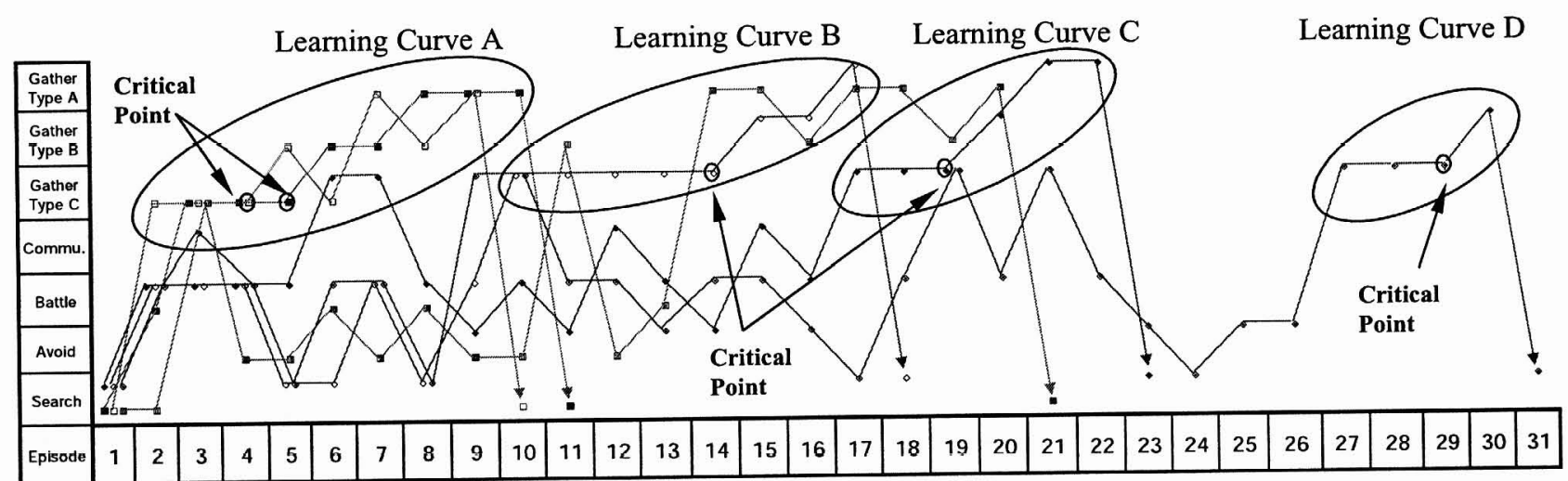

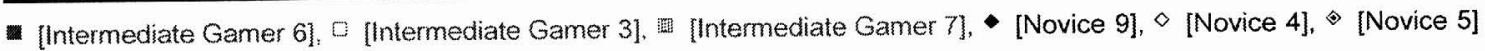

Critical points mean the transition from sequential-method to template-method.

Figure 2. Intermediate gamers v.s. Novics: The Summary of Play Behavior Graph

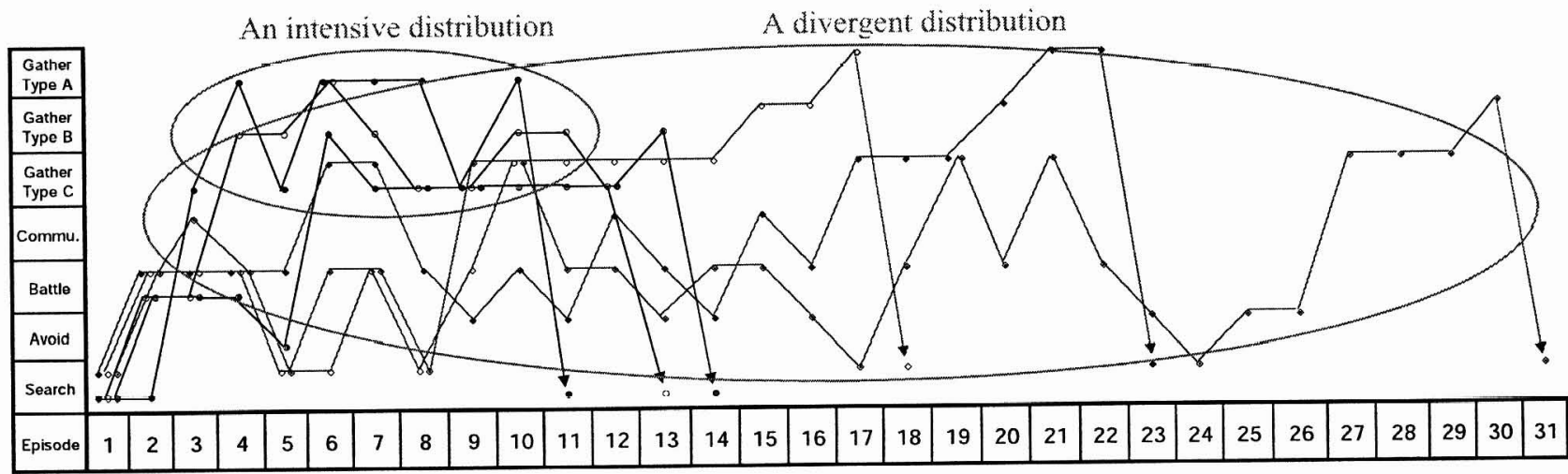

$\bullet$ [Novice 9], $\diamond[$ Novice 4], $\diamond[$ Novice 5], $\bullet$ [Expert 2], $\odot$ [Expert 8], 1 [Expert 1]

Figure 3. Novices v.s. Experts : The Summary of Play Behavior Graph 
similar learning curves because of being new to WOW game. However, there are more differences rather than the similarity between the two groups. The intermediate game players formed the learning curve from episode 2 to episode 10 in early stages of the game. However, the novices did so in the latter part of the game (episode $10 \sim 17,17 \sim 22$ and $27 \sim 30$ ). In addition, the intermediate game players understood the rule of games in the episode 4 and 5 to apply this rule to their gameplay. This means that they conduct the change of early stage from sequential-method ('Gather Type C') to the templatemethod ('Gather Type B'). However, novices applied this in the latter part of game like episode 14, 19 and 29 (see critical points in Figure 2). The behavior pattern was elucidated by much more time and steps in gameplay for novices than for intermediate gamers. Next, the intermediate gamers conducted 'gather action' as high frequency of use among functional actions. However, 'battle action' and 'search action' were performed more than 'gather action' for novices because they were found to refine a strategic knowledge to solve the constraints through 'battle action' and 'search action.' Furthermore, 'gather action' intensively appeared in the latter part of game for novices.

Figure 3 indicates the differences between experts and novices. While there was an intensive distribution in the early stage of the game for experts, the novices' behavior pattern appeared in a divergent distribution in the whole game. While novices showed the learning curves like intermediate gamers, there was nothing for experts. As previously mentioned, these differences were caused by the property of play. Therefore, experts' gameplay pattern seemed to have more feature of Ludus (rule) while novices' gameplay pattern seemed to have that of Paidea (free).

\section{Conclusion and discussion}

This research proposes an analytic framework for the gameplay. To this end, literatures related to gameplay were reviewed to conduct the empirical experiment in MMORPG where verbal and action protocols were collected. This study has investigated the comparison of experts, intermediate gamers, and novice.

This research has both strong theoretical and practical contributions. We found that the gameplay actions are definable in a systematic way using the vocabulary of the scheme, and thus a gamer's cognitive behaviors in each of the gameplay phases is represented as a structure composed of defined primitive actions. This is expected to lay the foundation for protocol analyses of how particular types of actions contribute to the formation of key design ideas. Consequently, theoretical contributions from this study will present a new analytic framework for the gameplay. Practical contributions of this research hold for applying the coherences and differences within each group to design guideline for the MMORPG game. Specifically, a concrete game planning is available because designers may determine the unit, the propensity, and the amount of consumption when a game feature is developed. Therefore, the results of this study will present the basis on the development of MMORPG game content. This is expected to lead us to the establishment of game design methodology for improving the quality of products.

This study examined the game players approximately for two hours in the game of WOW. Consequently, it is inadequate in explaining various patterns of the gameplay on 'Deep Play' with other races or classes. The behavior pattern of the latter part of the game will be studied in future research.

\section{References}

[1] C. B. John, and W. Mitchell, Got Game: How the Gamer Generation Is Reshaping Business Forever, Harvard Business Press, USA, 2004.

[2] D.Sheff, Game Over: Nintendo's Battle to Dominate an Industry, London: Hodder and Stoughton, 1993.

[3] G. Frasca, "Don't Play It Again, Sam: One-session and serial games of narration", Proceedings of digital arts \& culture, Bergen, Norway 1998.

[4] J. Newman, Videogames. Routledge: Taylor \& Francis Group, London, NY., 2004.

[5] ESA (Entertainment Software Association), 2005 Essential Facts About the Computer and Video Game at E3. 2005.

[6] J.B. Funk, "Reevaluating the impact of video games" Clinical Pediatrics, 32 (3), 1993, pp 86-90.

[7] G. Frasca, "Ludologists love stories, too: notes from a debate that never took place", Ludology.org. 2003.

[8] J. Huizinga, Homo Ludens: a Study of the play Element in Culture, Boston, MA: Beacon Press, 1955.

[9] R.Caillois, Man, Play and Games, Meyer Barash (trans.) Urbana, IL:University of Illiois Press, 1958.

[10] B. Bates, Game Design: The Art and Business of Creating Games, Roseville, GA: Prima Tech, 2001.

[11] A. Newell, and H. Simon, Human problem solving, Prentice Hall, Englewood Cliffs, New Jersey, 1972.

[12] M. Kavakli, and J. Thorne, "A Cognitive Modeling Approach to Capturing the context of complex Behavior in Gameplay", Proceedings of the First International Conference on Information Technology \& Applications (ICITA2002), IEEE, Bathurst, Australia, 25-29 November, 2002.

[13] M. Suwa, T. Purcell, and J. Gero, "Macroscopic analysis of design processes based on a scheme for coding designers' cognitive actions", Design Studies, vol 19, 1998, pp 455-483.

[14] J. Kim, F. Lerch, and H. Simon, "Internal Representation and Rule Development in Object-Oriented Design", $A C M$ Transactions on Computer-Human Interaction, 2(4), 1995, pp 357-390.

[15] B.E., John and A. Vera, "A GOMS Analysis of a Graphic, Machine-Paced, Highly Interactive Task", Proceedings of the conference on CHI , 1992, pp251-258.

[16] K. A. Ericcsson, and H. A. Simon, Protocol Analysis, MIT Press, Cambridge, MA, 1993. 